\title{
Remifentanil and sevoflurane based anesthesia combined with bilateral erector spinae plane block in patients undergoing off-pump coronary artery bypass graft surgery
}

\author{
Arkadiusz Kurowicki ${ }^{1}$, Michal Borys ${ }^{2}$, Slawomir Zurek ${ }^{1}$, Beata Horeczy ${ }^{3}$, Boguslaw Gaweda ${ }^{1}$, Bartlomiej Belina ${ }^{3}$, \\ Bozena Trojnar ${ }^{1}$, Bogumila Woloszczuk-Gebicka ${ }^{4}$, Justyna Sejboth ${ }^{5}$, Miroslaw Czuczwar ${ }^{2}$, Kazimierz Widenka ${ }^{1,4}$ \\ ${ }^{1}$ Clinical Department of Cardiac Surgery, St. Jadwiga Provincial Clinical Hospital, Rzeszow, Poland \\ $2^{2} 2^{\text {nd }}$ Department of Anaesthesiology and Intensive Care, Medical University of Lublin, Poland \\ ${ }^{3}$ Anesthesiology and Intensive Care Department with the Center for Acute Poisoning, St. Jadwiga Provincial Clinical Hospital, Rzeszow, \\ Poland \\ ${ }^{4}$ Medical College, Rzeszów University, Poland \\ ${ }^{5}$ Department of Anaesthesiology and Intensive Care, Department of Clinical Anaesthesiology, School of Health Sciences in Katowice, \\ Medical University of Silesia, Katowice, Poland
}

Videosurgery Miniinv 2020; 15 (2): 346-350 DOI: https://doi.org/10.5114/wiitm.2019.88748

\begin{abstract}
Introduction: Working on the institutional Enhanced Recovery After Surgery (ERAS Cardio) protocol for off-pump coronary artery bypass graft surgery (OP-CABG) we have noticed that patients treated according to the modified anesthesia protocol had not only significantly shorter time of respiratory support and intensive care unit stay but also lower postoperative troponin $T$ concentration than patients who had standard fentanyl/sevoflurane-based anesthesia.

Aim: To compare the perioperative course of patients undergoing OP-CABG surgery and receiving standard fentanyl/ sevoflurane anesthesia and those anesthetized according to the institutional ERAS Cardio protocol with remifentanil, sevoflurane, and bilateral extensor spinae plane (ESP) block.

Material and methods: Design: a prospective, open-label, observational study performed in a tertiary health center. Participants: 30 consecutive patients undergoing off-pump coronary bypass graft surgery. Interventions: 15 patients had standard anesthesia with etomidate, fentanyl, and rocuronium for induction and fentanyl/sevoflurane for maintenance (standard group); 15 others had bilateral single shot ESP block, then etomidate, remifentanil and rocuronium for induction, and remifentanil/sevoflurane for maintenance of anesthesia.

Results: Median time to extubation was $7.6(5.5-12.5) \mathrm{h}$ and $1.7(1-3.25) \mathrm{h}$ in "standard care" and ERAS groups, respectively $(p=0.00002)$. Length of stay in the intensive care unit was also shorter for patients in the ERAS group $20.5(18-24)$ vs. $48(42-48) h(p=0.00001)$. Troponin concentration increased to a lesser extent in patients from the ERAS group: an increase of $151.8(71.9-174.3) \mathrm{ng} / \mathrm{ml}$ vs. $253.8(126.6-373.1) \mathrm{ng} / \mathrm{ml}, p=0.008$.

Conclusions: Remifentanil/sevoflurane anesthesia combined with bilateral ESP block shortens mechanical ventilation time and ICU stay, and decreases postoperative troponin-T concentration in patients undergoing off-pump coronary bypass graft surgery.
\end{abstract}

Key words: fentanyl, remifentanil, troponin T, nerve block, coronary bypass grafting, off pump.

\footnotetext{
Address for correspondence

Bogumila Woloszczuk-Gebicka MD, PhD, Medical College, Rzeszow University, 6 Prof. Pigonia St, 35-310 Rzeszow, Poland,

phone: +48 602753 248, e-mail: gebicka@hotmail.com
} 


\section{Introduction}

Cardiac surgery is associated with a high complication rate and resource expenditure. In order to improve patients' outcomes, minimally invasive surgical techniques and novel enhanced recovery protocols are being introduced worldwide [1]. Coronary artery bypass surgery without the use of extracorporeal circulation (off-pump coronary artery bypass grafting - OP-CABG) is by default a minimally invasive method. It is also called aortic-coronary bypass surgery on a "beating heart". It was introduced to minimize morbidity and mortality associated with the use of extracorporeal circulation, aortic clamping, cardiac ischemia, induction of systemic inflammatory response, and activation of the coagulation system.

We have recently introduced an institutional Enhanced Recovery After Cardiac Surgery (ERAS Cardio) protocol, which includes bilateral erector spinae-plane (ESP) block as part of multimodal analgesia [2, 3], as well as the use of an extremely short acting opioid (remifentanil) instead of fentanyl during the surgery and in the early postoperative period. Some researchers claim that remifentanil might not be appropriate in the population of hemodynamically compromised patients [4]. Nevertheless, a recent meta-analysis did not confirm that speculation $[5,6]$. Our major goal was to shorten the duration of postoperative mechanical ventilation and speed up the recovery process without compromising patients' comfort and safety.

\section{Aim}

The aim of our study was to compare the perioperative course of patients undergoing OP-CABG surgery and receiving standard perioperative care and those treated according to the institutional ERAS Cardio protocol with remifentanil, sevoflurane and erector spinae plane block.

\section{Material and methods}

The study protocol was approved by the Bioethics Committee, and all patients signed informed consent for participation in the study.

Data from 30 consecutive patients undergoing OP-CABG surgery performed in a single tertiary health centre (teaching hospital) and participating in the prospective, open-label, observational study were analyzed. Fifteen patients had standard perioperative care ("standard group"), and the other 15 were treated according to institutional ERAS Cardio protocol (ERAS group). All procedures were performed by the same cardiac surgeon using the same minimally invasive technique (OP-CABG). With this technique, the anastomoses are created in the most physiological way, or "on the beating heart". It requires the use of a heart stabilizer (or stabilizers) and appropriate placement of the pericardial sutures. The use of a stabilizer allows for precise creation of the distal anastomosis, while pericardial sutures allow manipulation and proper positioning of the heart.

\section{Intervention}

\section{Anesthesia}

Fifteen patients from the ERAS group did not receive any pharmacological premedication. Midazolam at the dose of $7.5 \mathrm{mg}$ was administered orally in the "standard care" group.

In ERAS group, bilateral single shot ESP block was performed under ultrasound guidance with $0.375 \%$ ropivacaine $20 \mathrm{ml}$ per each side before the induction of general anesthesia.

In both groups, etomidate $0.2-0.3 \mathrm{mg} / \mathrm{kg}$ and rocuronium $0.6 \mathrm{mg} / \mathrm{kg}$ were used for general anesthesia induction and muscle relaxation, respectively. In the "standard care" group, a $2-4 \mu \mathrm{g} / \mathrm{kg}$ bolus dose of fentanyl was given at induction, followed by 25-100 $\mu \mathrm{g} / \mathrm{h}$ for maintenance. In the ERAS group, a $0.1-0.4 \mu \mathrm{g} / \mathrm{kg}$ bolus dose of remifentanil was administered at induction, followed by continuous infusion, 0.1-0.4 $\mu \mathrm{g} / \mathrm{kg} / \mathrm{min}$ (Perfusor, Braun, Germany, target-controlled infusion (TCI), target plasma concentration 4-8 $\mathrm{ng} / \mathrm{ml}$ ) for maintenance. 0.5 MAC of sevoflurane and incremental doses of rocuronium were used during maintenance of anesthesia in both groups.

All patients received $1.0 \mathrm{~g}$ of paracetamol intravenously (IV) at the end of surgery. Patients from the ERAS group also received $10 \mathrm{mg}$ of oxycodone, $8 \mathrm{mg}$ of dexamethasone and $8 \mathrm{mg}$ of ondansetron, all administered i.v. In the ERAS group, reduced remifentanil infusion ( $\mathrm{TCl}$, plasma concentration $0.5 \mathrm{ng} / \mathrm{ml}$ ) was continued for 1-2 $\mathrm{h}$ post-operatively, then stopped, and the patient's trachea was extubated. Patient-controlled analgesia (PCA) with oxycodone was provided immediately (1 $\mathrm{mg}$ per dose, lock-out time $7 \mathrm{~min}$, no basal infusion). An intravenous oxy- 
codone dose $(2 \times 5 \mathrm{mg})$ was available as rescue analgesia. In the "standard care" group, the trachea was extubated when the patient was awake, and postoperative pain relief was provided by nurses with incremental doses of morphine given at $0.1 \mathrm{mg} / \mathrm{kg}$ i.v.

Troponin T (high-sensitivity troponin $\mathrm{T}-\mathrm{hsTnT}$ ) was measured as a marker of myocardial injury a day before surgery and at 6 a.m. on the $1^{\text {st }}$ post-operative day with an Elecsys 2018 instrument (Roche Diagnostics, cobas e411 analyzer, cobas e601 module).

\section{Statistical analysis}

Data are reported as medians and interquartile ranges (IQR). Statistical analysis was performed using Statistica 13.1 (StatSoft. Inc., Tulsa, OK, USA). The Mann-Whitney test was used for paired comparisons. $P<0.05$ was considered statistically significant.

\section{Results}

Twenty-nine patients completed the study (26 male, 3 female). One patient from the ERAS group was excluded due to a complication leading to transient myocardial ischemia. Median age was 66 (IQR: 58-75) and 65.5 (IQR: 60-71) years, body mass index 27.7 (25-28.7) and 28.5 (27-31), Canadian Car-

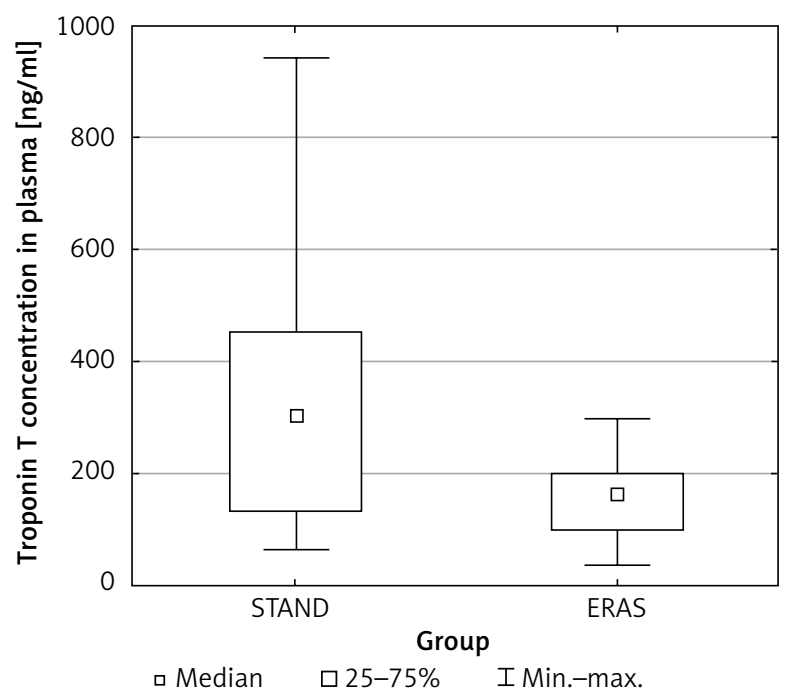

Figure 1. High-sensitivity troponin-T (hsTnT) concentration in plasma on the first post-operative day in patients receiving standard care or postoperative care compatible with the institutional ERAS Cardiac protocol diovascular Society (CCS) grading scale of angina pectoris was 3 (2-3) and 3 (2-3), NYHA (New York Heart Association) grading scale of heart failure was $1(1-2)$ and $1(1-2)$, and ASA (American Society of Anesthesiologists) Physical State group 3 (3-3) and 3 (3-3) in "standard care" and ERAS groups, respectively (NS).

Anesthesia time was 4.1 (4-5) $\mathrm{h}$ in the "standard care" and $4.1(3.2-4.2) h$ in the ERAS group $(p=0.2)$, and surgery time was $3.2(2.4-3.4) \mathrm{h}$ in the "standard care" group and $2.2(2.1-3.4) \mathrm{h}$ in the ERAS group $(p=0.16)$.

Median time to extubation of the patient's trachea was $7.6(5.5-12.5) \mathrm{h}$ and $1.7(1-3.25) \mathrm{h}$ in "standard care" and ERAS groups, respectively ( $p=$ $0.00002)$; therefore the principal objective of implementation of the ERAS Cardio protocol-shortening of respiratory support time - was achieved. The length of stay in the intensive care unit was also shorter for the patients in the ERAS group: 20.5 (18-24) vs. $48(42-48) \mathrm{h}(p=0.00001)$. Time to hospital discharge was 6 (5-7) days in ERAS group and $6(6-10)$ days in the "standard care" group ( $p=0.0507)$.

Preoperative hsTnT level was similar in "standard care" and "ERAS" groups: 13.59 (IQR: 8.12-27.4) $\mathrm{ng} / \mathrm{ml}$ and $11.1(9.88-26.15) \mathrm{ng} / \mathrm{ml}$, respectively, $p=0.81$ (NS), but postoperative troponin concentration taken in the morning of the first post-operative day was significantly lower in the ERAS group: 163.8 (103.3-198.7) $\mathrm{ng} / \mathrm{ml}$ vs. 305.4 (135.3-449.9) $\mathrm{ng} / \mathrm{ml}$ in "standard care" group, $p=0.0014$ (Figure 1). Troponin concentration in serum increased significantly less in patients in the ERAS group: an increase of 151.8 (71.9-174.3) $\mathrm{ng} / \mathrm{ml}$ compared with their counterparts from the "standard care" group: an increase of $253.8(126.6-373.1) \mathrm{ng} / \mathrm{ml}, p=0.008$ (Figure 2).

Neither postoperative troponin concentration nor the difference in troponin concentration between the post-operative and pre-operative value correlated with the number of grafts $\left(r^{2}=0.053\right.$ and 0.0012 , respectively).

Serum lactate concentration, urea, creatinine and electrolytes did not differ between study groups.

\section{Discussion}

This was a prospective, open-label, observational study aiming to compare the newly introduced ERAS Cardio protocol with the standard of care in patients undergoing OP-CABG. We have demonstrated several benefits associated with the use of the enhanced 
recovery pathway in patients undergoing OP-CABG surgery including significantly shorter time of mechanical ventilation in the postoperative period and the ICU stay, as well as lower postoperative troponin T concentrations.

General anesthesia with high doses of fentanyl seems to be the most commonly used analgesic option in a vast majority of cardiac patients worldwide [7-9]. Remifentanil, which is routinely used in ASA physical status groups III and IV, i.e. for bariatric surgery, is infrequently chosen for patients undergoing cardiac surgery even though some researchers claim that it may be beneficial in several ways $[10,11]$. In many patients anesthetized with high doses of fentanyl, the trachea can be extubated on the next day after surgery only, which seems to be related, at least in part, to the long context-sensitive half-life (CSHL) of fentanyl, and opioid-induced respiratory depression. Remifentanil is metabolized rapidly by plasma esterases, and its CSHL is 3 min only; therefore opioid-induced respiratory depression resolves almost immediately after discontinuation of remifentanil infusion, enabling early tracheal extubation and thus shorter ICU stay.

Regional techniques, such as epidural analgesia, are not performed routinely in patients undergoing cardiac surgery because of a potential risk of epidural hematoma formation due to systemic heparinization [12-14]. Bilateral continuous ESP block, as part of multimodal analgesia, has been shown to be effective for postoperative pain relief in cardiac patients undergoing sternotomy without such risk [13]. The decrease in time to tracheal extubation and ICU stay observed in the present study could also be associated with improved pain control provided by a regional anesthesia technique. A reduction in sympathetic nervous system activation in patients receiving bilateral ESP blocks compared with those who were not treated with regional anesthesia may partly explain the differences in high-sensitivity troponin $T$ concentrations between groups.

This study is a preliminary report only, and its obvious imitations are the small sample size and lack of randomization. Moreover, beside bilateral ESP block, patients in the ERAS Cardio group had a different opioid than their counterparts from the standard care group.

We hypothesize that the reduced intensity of painful stimuli from the operating site and a reduction in sympathetic nervous system activation in pa-

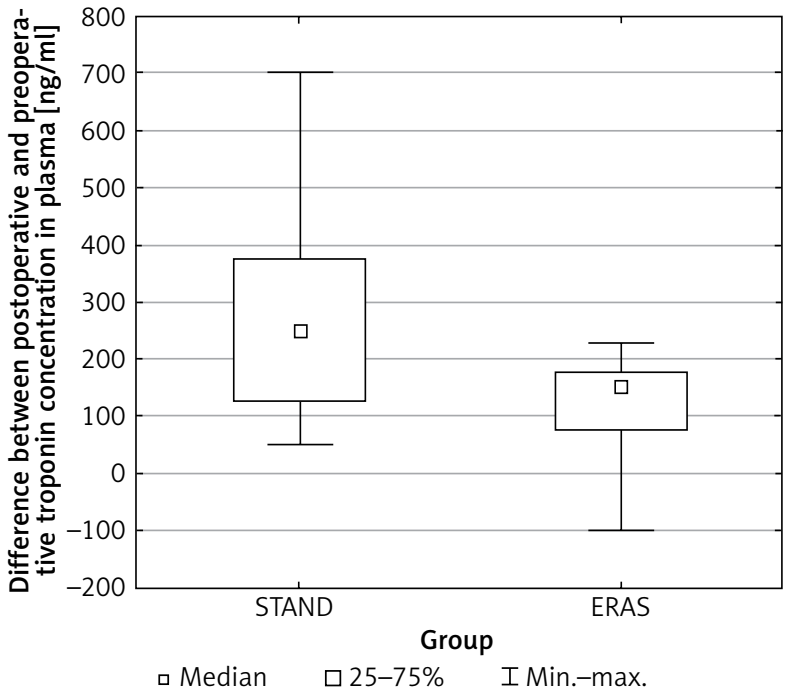

Figure 2. Postoperative increase of hsTnT concentration (the difference between postoperative and preoperative values) in patients receiving standard care or postoperative care compatible with the institutional ERAS Cardiac protocol

tients receiving remifentanil/sevoflurane anesthesia combined with the ESP blocks compared with those who had fentanyl/ sevoflurane anesthesia may contribute to the lower postoperative troponin T concentration. Measuring plasma norepinephrine and epinephrine concentrations is required to confirm this hypothesis, since lower concentration of catecholamines in plasma may explain the differences in troponin release between the study groups.

The applicability of the current findings requires further evaluation.

\section{Conclusions}

Modified perioperative treatment, using remifentanil/sevoflurane anesthesia combined with the bilateral ESP block, shortens the duration of mechanical ventilation, decreases postoperative troponin $\mathrm{T}$ concentration in plasma and shortens the ICU stay of patients undergoing OP-CABG surgery.

\section{Conflict of interest}

The authors declare no conflict of interest.

\section{References}

1. Noss C, Prusinkiewicz C, Nelson G, Patel PA, et al. Enhanced recovery for cardiac surgery. J Cardiothorac Vasc Surg 2018; 32: 2760-70. 
2. Forero M, Adhikary SD, Lopez $\mathrm{H}$, et al. The erector spinae plane block: a novel analgesic technique in thoracic neuropathic pain. Reg Anesth Pain Med 2016; 41: 621-7.

3. Borys M, Gawęda B, Horeczy B, et al. Erector spinae-plane block as an analgesic alternative in patients undergoing mitral and/ or tricuspid valve repair through a right mini-thoracotomy - an observational cohort study. Videosurgery Miniinv DOI: https:// doi.org/10.5114/wiitm.2019.85396.

4. Elliott P, O'Hare R, Bill KM, et al. Severe cardiovascular depres sion with remifentanil. Anesth Analg 2000; 91: 58-61.

5. Greco M, Landoni G, Biondi-Zocai G, et al. Remifentanil in cardiac surgery: a meta-analysis of randomized controlled trials. J Cardiothorac Vasc Anesth 2012; 26: 110-6.

6. Möllhoff T, Herregods L, Moerman A, et al. Comparative efficacy and safety of remifentanil and fentanyl in "fast track" coronary artery bypass graft surgery: a randomized, double blind study. $\mathrm{Br}$ J Anaesth 2001; 87: 718-26.

7. Myles PS, Hunt JO, Fletcher H, et al. Remifentanil, fentanyl, and cardiac surgery: a double-blinded, randomized, controlled trial of costs and outcomes. Anesth Analg 2002; 95: 805-12.

8. Kwanten LE, O'Brien B, Anwar S. Opioid-based anesthesia and analgesia for adult cardiac surgery: History and narrative review of the literature. J Cardiothorac Vasc Anesth 2019; 33: 808-16.

9. Williams JB, McConnell G, Allender JE, et al. One-year results from the first US-based enhanced recovery after cardiac surgery (ERAS Cardiac) program. J Thorac Cardiovasc Surg 2019 157: 1881-8.

10. Wong GT, Huang Z, Ji S, et al. Remifentanil reduces the release of biochemical markers of myocardial damage after coronary artery bypass surgery. A randomized trial. J Cardiothorac Vasc Anesth 2010; 24: 790-6.

11. Lee HJ, Tin TD, Kim YJ, et al. Remifentanil attenuates systemic inflammatory response in patients undergoing cardiac surgery with cardiopulmonary bypass. Arch Med 2017; 9: 5.

12. Kowalewski R, Seal D, Tang T, et al. Neuraxial anesthesia for cardiac surgery: thoracic epidural and high spinal anesthesiawhy is it different? HSR Proc Intensive Care Cardiovasc Anesth 2011; 3: 25-8.

13. Landoni G, Isella F, Greco M, et al. Benefits and risks of epidura analgesia in cardiac surgery. Br J Anaesth 2015: 115: 25-32.

14. Nagaraja PS, Ragavendran S, Singh NG, et al. Comparison of continuous thoracic epidural analgesia with bilateral erector spinae plane block for perioperative pain management in cardiac surgery. Ann Card Anaesth 2018; 21: 323-7.

Received: 6.07.2019, accepted: 16.09.2019. 